\title{
REVISED
}

\section{Multicenter evaluation of a real-time loop-mediated isothermal amplification(RealAmp) test for rapid diagnosis of Mycobacterium tuberculosis}

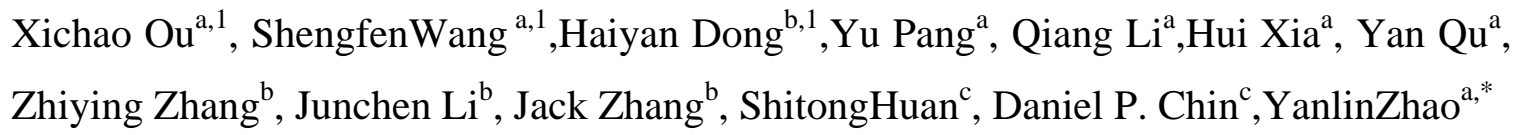

${ }^{a}$ National Center for Tuberculosis Control and Prevention, Chinese Center for Disease Control and Prevention, Beijing, P. R. China

${ }^{\mathrm{b}}$ PATH, China Office, Beijing, China

'Bill\& Melinda Gates Foundation, China Office, Beijing, China

*Corresponding author:

Yanlin Zhao, National Center for Tuberculosis Control and Prevention, Chinese Center for Disease Control and Prevention, No. 155, Changbai Road, Beijing. Fax 86-1058900777; E-mail:zhaoyanlin@chinatb.org.

${ }^{1}$ These authors contributed equally to this work.

\begin{abstract}
The real-time loop-mediated isothermal amplification (RealAmp) test is a novel technology for rapid diagnosis of Mycobacterium tuberculosis (MTB) from sputum samples. This test has not previously been effectively evaluated. In this study, we assessed the performance of the RealAmp test for tuberculosis (TB) detection among TB suspected patients from three provincial hospitals in China. Each eligible TB suspected patients in Shanghai, Guangzhou, and Shandong provided one sputum specimen each, smear microscopy, liquid culture, and the RealAmp test were performed per patient. A total of 1583 TB suspected patients were enrolled in the study, and 1519 were used for the performance analysis. The sensitivity and specificity of the RealAmp test for MTB
\end{abstract}


detection were calculated using liquid culture as reference method. The sensitivity of RealAmp test in smear-negative and culture-positive patients was $60.08 \%$; the sensitivity in smear-positive and culture-positive patients was $97.09 \%$; and the overall sensitivity in culture-positive patients was $74.88 \%$. The specificity of RealAmp test for MTB detection was $86.50 \%$.

\section{Keywords}

Mycobacterium tuberculosis, RealAmp, Sensitivity, Specificity

\section{Introduction}

Tuberculosis (TB) remains a global health problem. The World Health Organization (WHO) estimated that there were 9.0 million new TB cases and 1.5 million TB deaths in 2013. India and China accounted for $24 \%$ and $11 \%$ of total cases, respectively (World Health Organization, 2014).

Rapid and accurate diagnosis, followed by prompt treatment and completed therapy, is essential for effective control of TB (Small et al., 2010 and Pai et al., 2006). In most developing countries, TB diagnosis still depends on clinical examination, radiological findings, sputum microscopy, and bacterial culture (Urdea et al., 2006). Sputum smear microscopy is the most common method for screening TB worldwide because of its rapidity, simplicity, and cost-effectiveness (Steingart et al., 2007). However, it has limitations in sensitivity and the ability to differentiate Mycobacterium tuberculosis (MTB) complex (MTBC) from non-tuberculousis mycobacterium (NTM) (Kohet et al., 2006 and Luelmo et al., 2004).Culture is considered the gold standard method for diagnosis, with high sensitivity and specificity, but it usually takes 3 to 8 weeks get a result because of the slow growth rate of the mycobacterium (Small et al., 2010 and World Health Organization, 2004).

Following recent breakthroughs in TB diagnostics, the use of rapid molecular tests to diagnose TB is increasing (Pai et al., 2006, Helb et al., 2010 and Fang et al., 2009). Loopmediated isothermal amplification (LAMP) is a novel DNA amplification method that can be used in resource-limited settings (Notomi et al., 2000). Because simple equipment 
such as a water bath or an aluminum block bath is sufficient to accomplish the reaction in this test, more and more methods have been developed for MTB detection by LAMP (Adhikari et al., 2009, Pandey et al., 2008 and ou et al., 2014).However, the results of these methods are directly observed by the naked eye, which provides limited sensitivity compared with fluorescence-based LAMP.

The Mycobacterium tuberculosis DNA diagnostic kit developed by DEAOU Biotech Co., Ltd. (Guangzhou, China), employs a real-time loop-mediated isothermal amplification (RealAmp) method to amplify the target sequence of IS6110 under isothermal conditions. The amplification products emit florescent signals after reacting with nucleic acid dye (Figure 1). The Deaou isothermal amplification device uses modern micro-systems technology and the latest LED filtering processing technology. It can detect a signal and output the results according to the amplification curve. The device is a real-time fluorescence detection system that is highly efficient for the automatic detection of LAMP-generated products (Figure 2). The method consists of three steps: DNA extraction (50 $\mathrm{min})$, isothermal amplification (30min) and real-time detection (0.5-1 min). The speed of the reaction, the lack of need for a thermocycler, and the ease of use make the RealAmp test an excellent platform for molecular detection of MTB in developing countries.

This study was conducted in three provincial hospital laboratories to evaluate the performance of the RealAmp test for MTB detection on a greater scale.

\section{Materials and Methods}

\subsection{Study Design}

This study was conducted at three trial sites in China: Shandong Provincial Chest Hospital, Shanghai Pulmonary Hospital, and Guangzhou Chest Hospital. From June through August 2013, new TB suspected patients (cough and expectoration for at least 2 weeks, hemoptysis, and bloody sputum) who received less than two weeks TB treatment in last one month were consecutively recruited for the study. Each recruited case provided one spot sputum specimen of at least 3mL. Smear microscopy, MGIT culture, and RealAmp test were independently conducted by experienced personnel who were 
trained at the China National Reference Laboratory (NTRL) and who were blinded to the results of other tests. All culture-positive strains were transported to the NTRL for species identification (sequencing). The standard of care in these three hospital labs

comply with the guidelines for implementing the national tuberculosis control program in china.

\section{2 Procedures}

2.2.1Auramine O microscopy and MGIT culture: All sputum specimens were screened with auramine O microscopy in line with the China NTP-Sputum Smear Microscopy SOP and Quality Control Manual (Chinese Ministry of Health, 2009). Sputum specimens were also decontaminated using the NALC-NaOH method, and MGIT cultures were performed according to the manufacturer's instructions. All participating laboratories were quality-assured provincial reference laboratories. MGIT culture was routine work at each laboratory.

2.2.2 RealAmp test: The RealAmp test was performed on decontamination sputum specimens. (i) DNA extraction: Equal volume of $4 \% \mathrm{NaOH}$ is added to specimen for liquefying and decontamination. Vortex for $15 \mathrm{~s}$, place at room temperature for $15 \mathrm{~min}$, take $1 \mathrm{ml}$ supernatant into screw-cap tube. Centrifuge at $10000 \times \mathrm{g}$ for $5 \mathrm{~min}$, discard the supernatant. Add $1 \mathrm{ml}$ saline and mix well, centrifuge at $10000 \times \mathrm{g}$ for $5 \mathrm{~min}$, discard the supernatant. Add $100 \mu \mathrm{l}$ DNA extraction reagent, vortex for $15 \mathrm{~s}$. Heat at $100^{\circ} \mathrm{C}$ for 10 min. Centrifuge at $10000 \times \mathrm{g}$ for $2 \mathrm{~min}$, transport the supernatant into a new tube, $2 \mu 1$ supernatant for amplification. (ii) Reagent prepare: Take $25 \mu 1$ Bst polymerase into the "Reaction mixture", mix well, distribute $23 \mu \mathrm{l}$ mixture each tube into $0.2 \mathrm{ml}$ PCR tube (two more tube for positive and negative control). (iii) Add samples: Add $20 \mu 1$ sealing oil into the PCR tube, then add $2 \mu \mathrm{l}$ negative control, samples and positive control, respectively, centrifuge at $620 \times \mathrm{g}$ for 30 s. (iv) Amplification: $63^{\circ} \mathrm{C}$ for $30 \mathrm{~min}$ in a Deaou isothermal amplification device (designed in Germany, made in China) (Fig. 2). (v) Analysis of results: "S" shape amplification curves mean positive amplification otherwise negative amplification (Fig. 1). 
2.2.3 Species identification: All positive cultures were transported to NTRL, 16S-23S rDNA ITS sequence analysis was performed using a forward primer, 5'GGCCTAACCCTCGGGAGGGAG-3', and reverse primer 5'CCCGAGGCATATCGCAGCCTC-3' (Gürtler, 1996). Primers were synthesized by Sangon Biotech Co., Ltd (Shanghai, China).Direct sequencing was performed on the ABI 3730 DNA automated sequence analyzer. The sequencing results were entered into the Basic Local Alignment Search Tool (BLAST), an international data bank (www.ncbi.nlm.nih.gov/BLAST), and compared against the sequences of reference mycobacterium strains.

\subsection{Statistical analysis}

Using MGIT culture as the reference method, we calculated the sensitivity, specificity, positive and negative predictive values (PPV and NPV) by using SPSS 19.0 software.

\section{Results}

\subsection{Study population}

Between June and August 2013, 1583 eligible TB suspected patients were recruited for the study. For analysis, 64 cases were excluded because of culture contamination, no sequence result or NTM infection. Thus, 1519 TB suspected patients remained for performance analysis (Figure 3). Among these patients, 958 were male, and 106 were 20 years old or younger. Also, 172 were smear-positive and culture-positive TB cases, 258 were smear-negative and culture-positive TB cases, and 1089 were culture-negative TB cases (Table 1).

\subsection{Quality control of MGIT culture and RealAmp test}

A total of 1583 sputum specimens were cultured, and sixty-five samples were contaminated, for a contamination rate of $4.1 \%$. The smear-positive and culture-negative rate was $6.8 \%$ (14/205), which was below the standard operation procedure (SOP) requirement of $10 \%$.

277 runs of RealAmp test were performed, and four runs affected by false-positive negative controls, resulting in an indeterminate rate of $1.4 \%$ (4/277). 


\subsection{Results of 16-23s rDNA ITS sequence analysis for culture-positive strains}

16-23s rDNA ITS sequence analysis was performed on 491 culture-positive strains. 11 samples have no reported sequencing results. Among the 480 samples with successful sequencing results, $89.6 \%$ (430/480) were identified as MTB and $10.4 \%(50 / 480)$ as NTM. Among the 50 NTM-infected patients, 13 were smear-positive and 5 were RealAmp-positive.

\subsection{Performance of RealAmp test for MTB detection}

Among the 1519 analyzed TB suspected patients, the sensitivity and specificity of the RealAmp test for MTB detection were calculated using MGIT culture as the reference method (Table 2). The diagnostic results for1264 TB suspected patients (83.2\%) were consistent with those determined by MGIT culture. The sensitivity for MTB detection in smear-positive TB patients was $97.1 \%$, and the sensitivity for MTB detection in smearnegative TB patients was $60.1 \%$. The specificity for MTB detection was $86.5 \%$. The PPV and NPV for MTB detection was $68.7 \%$ and $89.7 \%$, respectively.

\section{Discussion}

Early and rapid diagnosis is very important for TB control. The RealAmp test is a novel, simple, LAMP based and real-time technology for the detection of MTB, the RealAmp system could be used in an electricity-free environment (the device could be battery operated). In addition, the cost of the instrument and the unit price of the RealAmp test are lower than costs for culture and other molecular tests. The cost per test of the RealAmp test is approximately $\$ 7-\$ 8$. All of these features make it a promising tool for use in developing countries.

Previous studies have demonstrated that the prevalence of NTM in China has increased significantly in recent years. According to the fifth national tuberculosis survey in 2010, the proportion of NTM in China was 22\% (Wang et al., 2014). In this study, we found that the prevalence of NTM in these TB specialized hospitals was 3.2\% (50/1583). One possible explanation for this difference may be that the national survey included all residents rather than just TB suspected patients. Hence, the NTMs, opportunistic pathogens, may be cultured and identified by laboratories, thereby resulting in the higher 
prevalence of NTM. The increasing prevalence of NTM highlights the urgent need to perform species identification to eliminate potential interference in detecting $\mathrm{TB}$ in clinical trials conducted in China.

We found that 5 out of 50 NTM cases were positive by RealAmp test, this may result from mixed infection with MTB that solid culture media rather than liquid media could differentiate, for the overgrowth by bacteria or NTM could suppress the growth of MTB in the liquid culture media occasionally, however, it is generally agreed that mixed infections are rare. The sensitivity of the RealAmp test in smear-positive and smearnegative patients was $97.1 \%$ and $60.1 \%$, respectively, similar to results in other studies (Ou et al., 2014 and Li et al., 2014).But our results showed lower specificity and PPV, possibly due to the possibility that RealAmp can detect uncultured or dead bacteria, but liquid culture could only detect live bacteria. Harsh specimen decontamination procedures, such as the pretreatment time more than $20 \mathrm{~min}$ or the ratio of pretreatment solution and specimen volume was more than two, will harmful for mycobacterium growth and decreased the sensitivity of MGIT culture. Among the 1089 culture-negative samples, 147 were RealAmp positive. Among the 147 culture-negative but RealAmppositive patients, 36 were clinically diagnosed non-TB. These false positives may have been due to the operation bias or nonspecific amplification of RealAmp test. Because the PPV is also influenced by TB prevalence, given equal sensitivity and specificity, lower prevalence will leads to lower positive predictive values, too.

There were two obvious limitations in this study. First, this evaluation was carried out in three provincial hospitals with high resource settings. Hence, the data of this study may not be representative of laboratories in resource-limited regions. Furthermore, the gold standard used herein was liquid culture rather than solid culture, which may lead to the missed detection of MTB because of mixed MTB/NTM infections.

In conclusion, the study results indicate that the RealAmp test has potential as a rapid test for TB diagnosis. Recently, WHO has published Target Product Profile (TPP) for Rapid sputum-based test for detecting TB at the microscopy-center level of the health-care system, while the sensitivity and specificity of RealAmp test in smear positive patients was lower than the requirements ( $99 \%$ for sensitivity and $98 \%$ for specificity), indicating 
that the modification of RealAmp test is urgently needed to improve the performance in the future.

\section{Ethics}

This study was reviewed and approved by the ethics review board of the Chinese Center for Disease Control and Prevention. Because the RealAmp test results were not used in clinical decision making, so the requirement to obtain individual informed consent was waived by the review board.

\section{Acknowledgments}

We gratefully thank the Bill \& Melinda Gates Foundation for its financial supportand PATH for its assistance in implementing the project, analyzing data, and writing this paper. We also thank all staff in project sites that contributed to this work.

\section{References}

Global tuberculosis report. Geneva, Switzerland: World Health Organization; 2014.

Small PM, Pai M. Tuberculosis diagnosis - time for a game change. N Engl J Med 2010; 363: 1070-1071.

Pai M, Kalantri S, Dheda K. New tools and emerging technologies for the diagnosis of tuberculosis: part II. Active tuberculosis and drug resistance. Expert Rev MolDiagn2006; 6: $423-432$.

Urdea M, Penny LA, Olmsted SS, Giovanni MY, Kaspar P, Shepherd A, Wilson P, Dahl CA, Buchsbaum S, Moeller G, Hay Burgess DC. Requirements for high impact diagnostics in the developing world. Nature2006; 23; 444: Suppl 1:73-79.

Steingart KR, Ramsay A, Pai M. Optimizing sputum smear microscopy for the diagnosis of pulmonary tuberculosis. Expert Rev Anti Infect Ther2007; 5:327-331.

Koh WJ, Yu CM, Suh GY, Chung MP, Kim H, Kwon OJ, Lee NY, Chung MJ, Lee KS. Pulmonary TB and NTM lung disease: comparison of characteristics in patients with AFB smear-positive sputum. Int J Tuberc Lung Dis2006; 10(9):1001-1007. 
Luelmo F. What is the role of sputum microscopy in patients attending health facilities? In: Toman's Tuberculosis: Case Detection, Treatment, and Monitoring-Questions and Answers. Frieden TR(Ed.). 2004; World Health Organization, Geneva. Switzerland: 7-10. Van Deun A. What is the role of mycobacterial culture in diagnosis and case definition? In: Toman's Tuberculosis: Case Detection, Treatment, and Monitoring-Questions and Answers. Frieden TR(Ed.). 2004; World Health Organization, Geneva. Switzerland 35-43. Pai M, Kalantri S, and Dheda K. New tools and emerging technologies for the diagnosis of tuberculosis: Part II. Active tuberculosis and drug resistance. Exp Rev MolDiagn2006; 6:423-432.

Helb D, Jones M, Story E, Boehme C, Wallace E, Ho K, Kop J, Owens MR, Rodgers R, Banada P, Safi H, Blakemore R, Lan NT, Jones-López EC, Levi M, Burday M, Ayakaka I, Mugerwa RD, McMillan B, Winn-Deen E, Christel L, Dailey P, Perkins MD, Persing DH, Alland D. Rapid detection of Mycobacterium tuberculosis and rifampin resistance by use of ondemand, near-patient technology.J ClinMicrobiol2010;48:229-237.

Fang R, Li X, Hu L, You Q, Li J, Wu J, Xu P, Zhong H, Luo Y, Mei J, Gao Q. CrossPriming Amplification for Rapid Detection of Mycobacterium tuberculosis in Sputum Specimens. J ClinMicrobiol2009; 47: 845-847.

Notomi T, Okayama H, Masubuchi H, Yonekawa T, Watanabe K, Amino N, Hase T. Loop-mediated isothermal amplification of DNA. Nucleic Acids Res 2000; 28: 63-63.

Adhikari BR, Pandey BD, Ghimire P, Shrestha B, Khadka M, Yoda T, Suzuki Y. Loopmediated isothermal amplification (LAMP) for the direct detection of human pulmonary infections with environmental (nontuberculosis) mycobacteria. Jpn J Infect Dis 2009; 62: $212-214$.

Pandey BD, Poudel A, Yoda T, Tamaru A, Oda N, Fukushima Y, Lekhak B, Risal B, Acharya B, Sapkota B, Nakajima C, Taniguchi T, Phetsuksiri B, Suzuki Y. Development of an in-house loop-mediated isothermal amplification (LAMP) assay for detection of Mycobacterium tuberculosis and evaluation in sputum samples of Nepalese patients. J Med Microbiol2008; 57: 439-443. 
Ou X, Li Q, Xia H, Pang Y, Wang S, Zhao B, Song Y, Zhou Y, Zheng Y, Zhang Z, Zhang Z, Li J, Dong H, Zhang J, Kam KM, Chi J, Huan S, Chin DP, Zhao Y. Diagnostic Accuracy of PURE-LAMP test for Pulmonary Tuberculosis at the County Level laboratory in China. PLOS ONE, 2014; 9(5):e94544.

Li Y, Shi L, Pan A, Cao W, Chen X, MengH, Yan H, Miyoshi S, Ye L. Evaluation of real-time loop-mediated isothermal amplification (RealAmp) for rapid detection of Mycobacterium tuberculosis from sputum samples. J Microbiol Methods2014; 104:55-58.

Chinese Ministry of Health, Disease Control and Prevention Bureau, Center for Disease Control and Prevention China TB control program. Sputum smear microscopy SOP and quality control manual. 2009, Beijing, China.

Gürtler V, Stanisich VA. New approaches to typing and identification of bacteria using the 16S-23S rDNA spacer region. Microbiology1996, 142: 3-16.

Wang L, Zhang H, Ruan Y, Chin DP, Xia Y. Tuberculosis prevalence inChina, 19902010; a longitudinal analysis of national survey data.Lancet 2014; 383(9934):2057-2064. 
Table 1 Demographic information for the participants who were analyzed

\begin{tabular}{|c|c|c|c|c|}
\hline \multirow{2}{*}{ Variable } & $\begin{array}{l}\text { Shandong } \\
(N=519)\end{array}$ & \multirow{2}{*}{$\begin{array}{l}\text { Shanghai }(N=477) \\
\text { No. }(\%)\end{array}$} & \multirow{2}{*}{$\begin{array}{l}\text { Guangzhou }(N=523) \\
\text { No. }(\%)\end{array}$} & \multirow{2}{*}{$\begin{array}{l}\text { Total }(N=1519) \\
\text { No. }(\%)\end{array}$} \\
\hline & & & & \\
\hline \multicolumn{5}{|l|}{ Age range } \\
\hline$<20$ & $42(8.09)$ & $23(4.82)$ & $41(7.84)$ & $106(6.98)$ \\
\hline $20-39$ & 197 (37.96) & $178(37.32)$ & $179(34.23)$ & $554(36.47)$ \\
\hline $40-59$ & $135(26.01)$ & $136(28.51)$ & $178(34.03)$ & 449 (29.56) \\
\hline$\geqslant 60$ & 145 (27.94) & $140(29.35)$ & $125(23.90)$ & $410(26.99)$ \\
\hline \multicolumn{5}{|l|}{ Gender } \\
\hline Female & 199 (38.34) & $173(36.27)$ & $189(36.14)$ & $561(36.93)$ \\
\hline Male & $320(61.66)$ & $304(63.73)$ & 334 (63.86) & $958(63.07)$ \\
\hline \multicolumn{5}{|l|}{ Culture result } \\
\hline $\begin{array}{l}\text { Smear- } \\
\text { positive/culture- } \\
\text { positive }\end{array}$ & $53(10.21)$ & 57 (11.95) & $62(11.85 \%)$ & $172(11.32)$ \\
\hline Smear- & & & & \\
\hline $\begin{array}{l}\text { negative/culture- } \\
\text { positive }\end{array}$ & 87 (16.76) & $52(10.90)$ & $119(22.75)$ & $258(16.98)$ \\
\hline Culture-negative & $379(73.03)$ & $368(77.15)$ & $342(65.39)$ & $1089(71.69)$ \\
\hline
\end{tabular}


Table 2 Performance of RealAmp test for MTB detection

\begin{tabular}{|c|c|c|c|c|c|c|}
\hline \multirow{2}{*}{$\begin{array}{c}\text { Site and no. } \\
\text { of test }\end{array}$} & \multicolumn{3}{|c|}{ Sensitivity $(95 \% \mathrm{CI})$} & \multirow{2}{*}{$\begin{array}{l}\text { Specificity } \\
(95 \% \mathrm{CI})\end{array}$} & \multirow{2}{*}{$\begin{array}{c}\text { PPV } \\
(95 \% \mathrm{CI})\end{array}$} & \multirow{2}{*}{$\begin{array}{c}\text { NPV } \\
(95 \% \mathrm{CI})\end{array}$} \\
\hline & $\begin{array}{l}\text { Smear- } \\
\text { culture+ }\end{array}$ & $\begin{array}{l}\text { Smear+ } \\
\text { culture+ }\end{array}$ & Culture + & & & \\
\hline \multirow{3}{*}{ Shandong } & $55.17 \%$ & $98.11 \%$ & $71.43 \%$ & $81.79 \%$ & $59.17 \%$ & $88.57 \%$ \\
\hline & $(48 / 87)$ & $(52 / 53)$ & $(100 / 140)$ & $(310 / 379)$ & $(100 / 169)$ & $(310 / 350)$ \\
\hline & $(44.72-65.18)$ & (90.06-99.67) & $(63.45-78.26)$ & $(77.60-85.35)$ & $(51.64-66.3)$ & $(84.81-91.49)$ \\
\hline \multirow{3}{*}{ Shanghai } & $75.00 \%$ & $94.74 \%$ & $85.32 \%$ & $84.78 \%$ & $62.42 \%$ & $95.12 \%$ \\
\hline & $(39 / 52)$ & $(54 / 57)$ & $(93 / 109)$ & $(312 / 368)$ & $(93 / 149)$ & $(312 / 328)$ \\
\hline & $(61.79-84.77)$ & $(85.63-98.19)$ & $(77.48-90.76)$ & $(80.75-88.09)$ & $(54.42-69.79)$ & $(92.22-96.98)$ \\
\hline \multirow{3}{*}{ Guangzhou } & $57.14 \%$ & $98.39 \%$ & $71.27 \%$ & $93.57 \%$ & $85.43 \%$ & $86.02 \%$ \\
\hline & $(68 / 119)$ & $(61 / 62)$ & $(129 / 181)$ & $(320 / 342)$ & $(129 / 151)$ & $(320 / 372)$ \\
\hline & $(48.17-65.67)$ & $(91.41-99.71)$ & (64.29-77.37) & $(90.45-95.71)$ & (78.93-90.18) & $(82.13-89.18)$ \\
\hline \multirow{3}{*}{ Total } & $60.08 \%$ & $97.09 \%$ & $74.88 \%$ & $86.50 \%$ & $68.66 \%$ & $89.71 \%$ \\
\hline & $(155 / 258)$ & $(167 / 172)$ & $(322 / 430)$ & $(942 / 1089)$ & $(322 / 469)$ & $(942 / 1050)$ \\
\hline & $(54-65.86)$ & $(93.38-98.75)$ & $(70.58-78.75)$ & $(84.34-88.40)$ & $(64.32-72.69)$ & $(87.73-91.40)$ \\
\hline
\end{tabular}




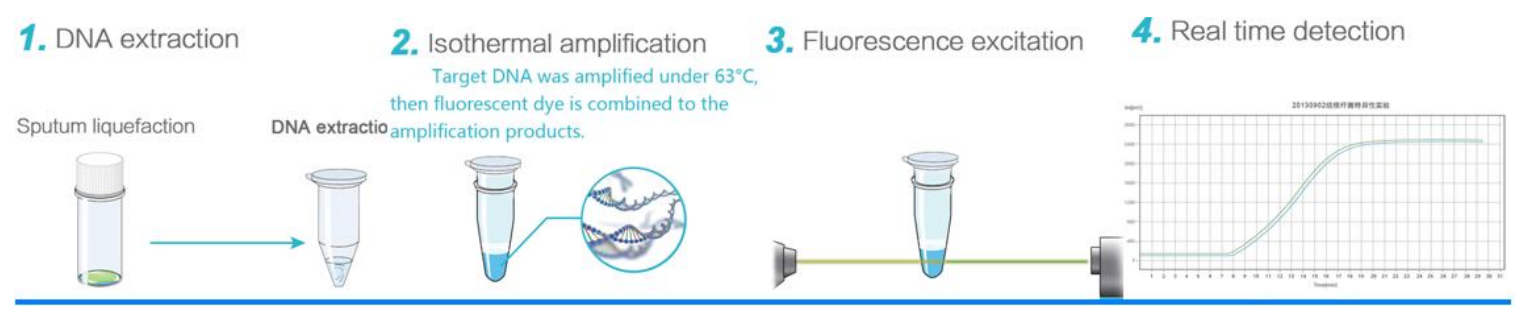

Figure 1 Schematic diagram for the RealAmp procedural steps

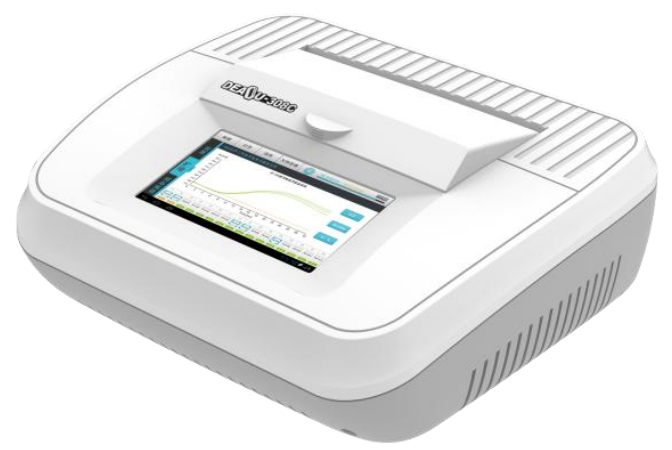

Figure 2 Deaou isothermal amplification device for RealAmp test

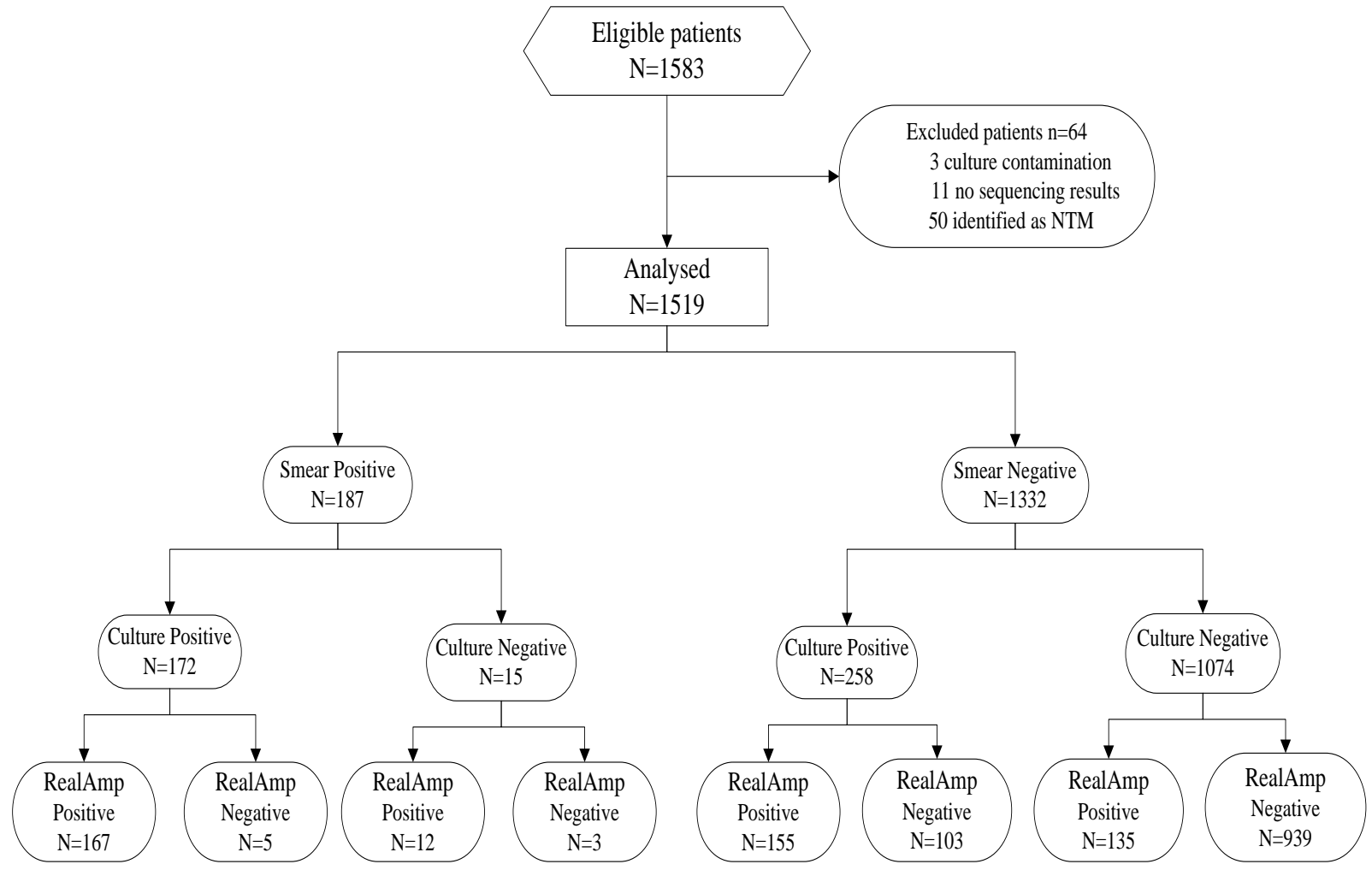

Figure 3 Flow chart for patient enrollment and testing 


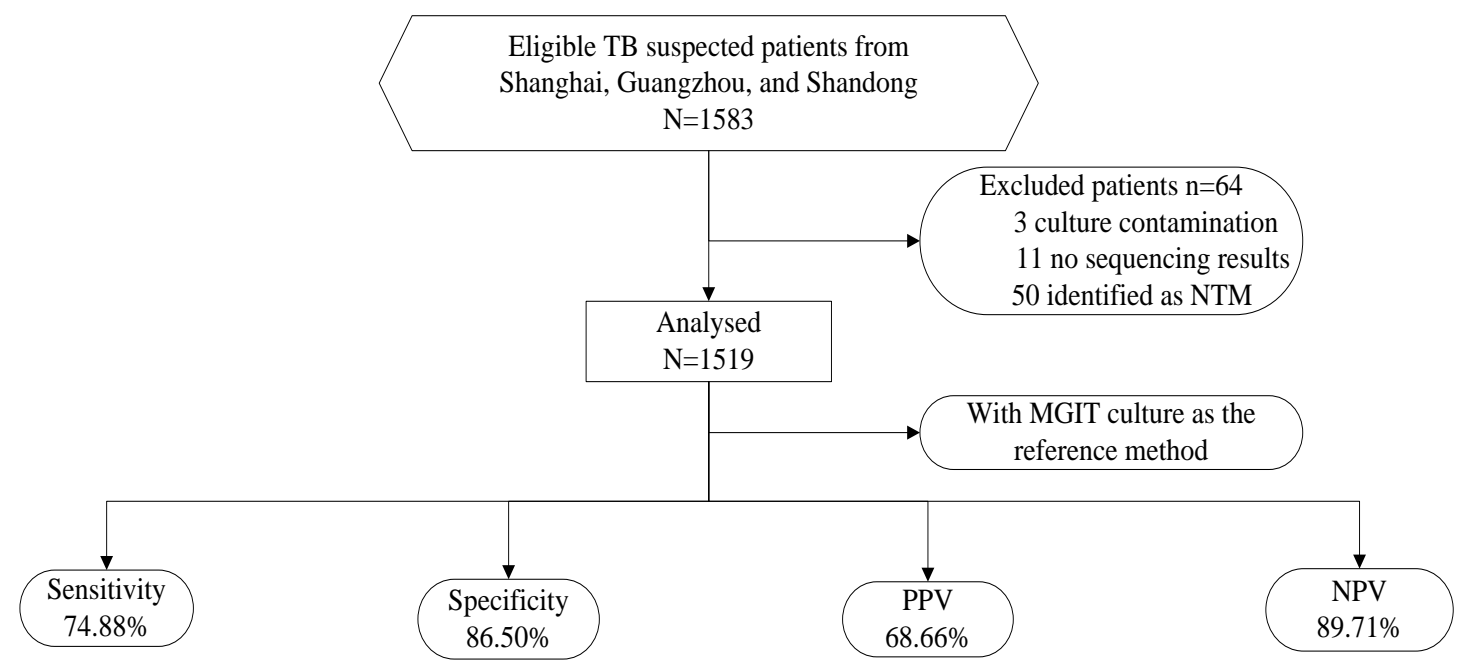

Graphical Abstract 